\title{
In vivo label-free photoacoustic microscopy of the anterior segment of the mouse eye
}

Bin Rao, Song Hu, Li Li, Konstantin Maslov, Lihong V. Wang

Bin Rao, Song Hu, Li Li, Konstantin Maslov, Lihong V. Wang, "In vivo labelfree photoacoustic microscopy of the anterior segment of the mouse eye," Proc. SPIE 7564, Photons Plus Ultrasound: Imaging and Sensing 2010, 75643E (23 February 2010); doi: 10.1117/12.843052

SPIE. Event: SPIE BiOS, 2010, San Francisco, California, United States 


\title{
In vivo, Label-free Photoacoustic Microscopy of the Anterior Segment of the Mouse Eye
}

\author{
Bin Rao, Song Hu, Li Li, Konstantin Maslov and Lihong V. Wang \\ Optical Imaging Laboratory, Department of Biomedical Engineering, Washington University in St. \\ Louis, St. Louis, MO 63130
}

\begin{abstract}
Both iris fluorescein angiography (IFA) and indocyanine green angiography (ICGA) provide ophthalmologists imaging tools in studying the microvasculature structure and hemodynamics of the anterior segment of the eye in normal and diseased status. However, a non-invasive, endogenous imaging modality is preferable for the monitoring of hemodynamics of the iris microvasculature. We investigated the in vivo, label-free ocular anterior segment imaging with photo-acoustic microscopy (PAM) in mouse eyes. We demonstrated the unique advantage of endogenous contrast that is not available in both IFA and ICGA. The laser radiation was maintained within the ANSI laser safety limit. The in vivo, label-free nature of our imaging technology has the potential for ophthalmic applications.
\end{abstract}

Keywords: photoacoustic microscopy, anterior segment of eye, iris imaging

\section{INTRODUCTION}

Iris fluorescein angiography (IFA) is a valuable technique for elucidating a wide range of eye pathologies such as developmental anomalies, degenerative disease, retinal vascular occlusions, diabetic microangiopathy, uveitis of various etiologies, glaucoma, tumor, and surgical or accidental trauma. IFA's clinic applications include the early detection of iris neovasculation (Rubeosis Iridis), cataract management in diabetic patients, and monitoring of iris tumors [1]. Indocyanine green angiography (ICGA) combined in a scanning laser ophthalmoscope uses deeper penetration infrared light to overcome some limitations of IFA [2]. Both IFA and ICGA provide ophthalmologists imaging tools in studying the microvasculature structure and hemodynamics of the anterior segment of eye in normal and diseased status. However, they are not widely used as mainstream diagnostic instruments in eye clinics due to multiple reasons. One major reason might be the invasive nature of IFA and ICGA. The recent development of optical-resolution photoacoustic microscopy (OR-PAM) [3], based on detecting the optical absorption contrast mechanism, introduces a novel member into the family of modern optical microscopy. OR-PAM, along with dark-field acoustic-resolution photoacoustic microscopy (AR-PAM), photoacoustic computed tomography and thermoacoustic tomography, promises to have a substantial impact on biomedical applications in the near future $[4,5]$. In this communication, we proposed and demonstrated an in vivo, label-free imaging of the anterior segment of the mouse eye with our OR-PAM technology.

Two types of OR-PAM instruments are used for demonstration. The original OR-PAM instrument [3] was used for the first OR-PAM iris imaging demonstration [6]. In the mean time, we developed another fast imaging speed, hybrid-scan OR-PAM (HSOR-PAM) instrument [7] to avoid the speed limitation of the original OR-PAM. Both imaging instruments demonstrated in vivo, label-free photoacoustic microscopy of the anterior segment of mouse eyes. The maximum permissible laser pulse energy was calculated according to the ANSI laser safety standard [8]. The laser radiation used in our experiments was within the ANSI laser safety limit.

Photons Plus Ultrasound: Imaging and Sensing 2010, edited by Alexander A. Oraevsky, Lihong V. Wang, Proc. of SPIE Vol. 7564, 75643E · (C) 2010 SPIE · CCC code: 1605-7422/10/\$18 · doi: 10.1117/12.843052 


\section{Materials and methods}

Schematic OR-PAM setup for ocular anterior segment imaging [6]

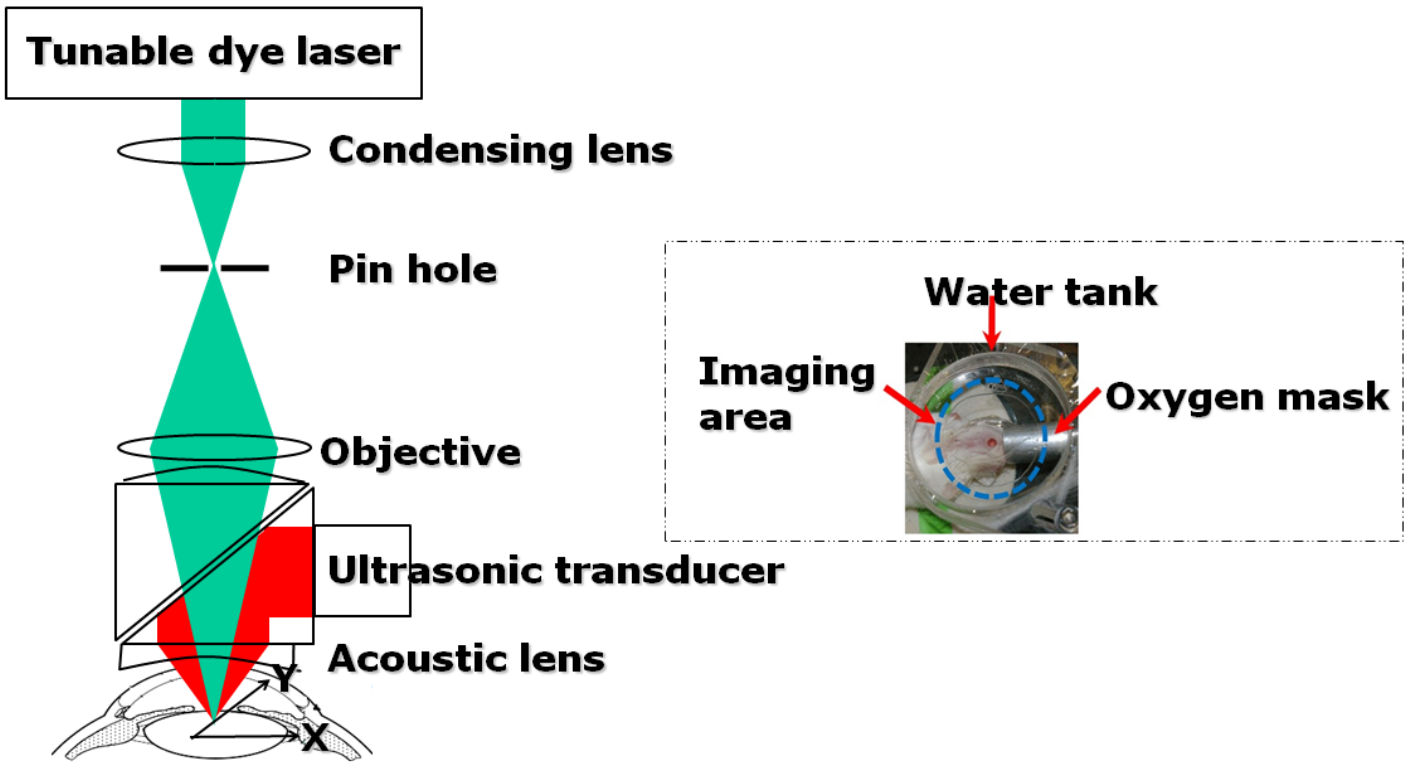

\section{Animal moved by 2D stages}

Fig. 1. Schematic setup of the optical-resolution photoacoustic microscopy (OR-PAM) system

As show in Figure 1, the collimated laser beam from a tunable dye laser was focused through a pin hole for spatial mode shaping. The pin hole was imaged onto the ocular anterior segment tissue through an objective lens (NA 0.1), a homemade plane-convex lens, two silica prisms, and a plane-concave lens (acoustic lens). The silicone oil embedded between two silica prisms was used to reflect ultrasonic wave to an ultrasonic transducer for signal detection. The dashed box in Figure 1 showed the animal position for imaging acquisition. The water tank shown in the dashed box was neglected in the schematic figure to avoid complexity. In order to maximize SNR, the image of the pin hole was designed to be at the center of the acoustic lens focus.

Schematic HSOR-PAM setup for ocular anterior segment imaging [7]

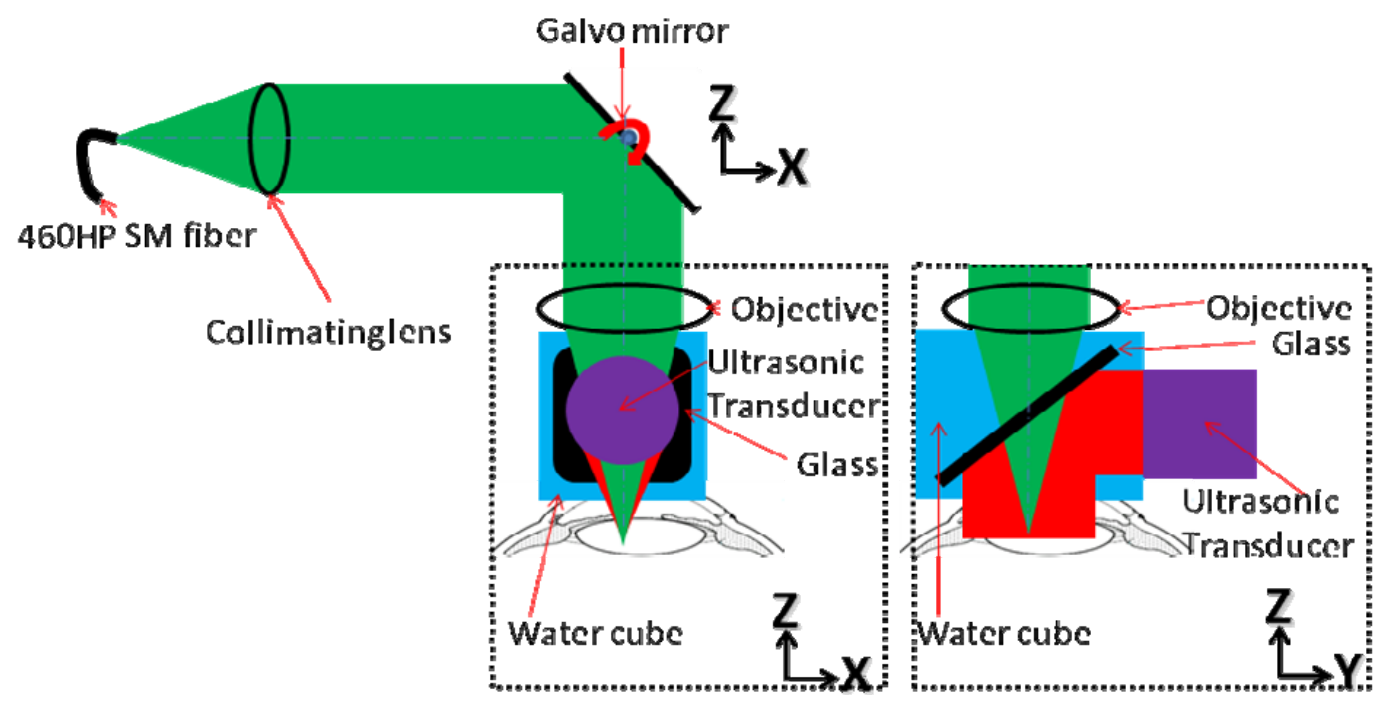

Fig. 2. Schematic setup of the hybrid-scan optical-resolution photoacoustic microscopy (HSOR-PAM) system 
Figure 2 is the schematic setup of the HSOR-PAM system. As shown in Figure 2, the excitation laser (532 nm) was delivered to the imaging system through a $460 \mathrm{HP}$ single mode fiber. The laser beam was collimated before being deflected by a Galvo mirror. The Galvo mirror was located at the front focus of an objective (NA 0.1). A water cube comprising two isosceles right-angle water prisms and a glass plate between them was employed for light wave delivery and acoustic wave coupling. After passing through the water cube, the laser beam was focused at least $200 \mu \mathrm{m}$ below the tissue surface. Upon the absorption of the laser pulse by the tissue, photoacoustic waves were generated. Thereafter, it was reflected by the glass plate and detected by the ultrasonic transducer. The ultrasonic transducer (GE, $25 \mathrm{MHz}$ bandwidth), having a line focus along the optical scan trace, collected photoacoustic signals from the fast optical scan axis ( $\mathrm{X}$ axis) without being moved mechanically. The whole probe was attached to a one-dimensional mechanical stage, which scanned perpendicularly to the fast optical scan axis.

\section{Imaging procedures}

All experimental animal procedures were carried out in conformity with the laboratory animal protocol approved by the School of Medicine Animal Studies Committee of Washington University in St. Louis. Nude mice (Hsd, Athymic Nude-Foxn $1^{\mathrm{NU}}$, Harlan Co.) were anesthetized by intraperitoneal (IP) injection of a cocktail containing Ketamine (100 $\mathrm{mg} / \mathrm{kg}$ ) and Xylazine $(10 \mathrm{mg} / \mathrm{kg})$. A custom-made animal holder was used to restrain the animal. A breathing device (E$\mathrm{Z}$ Anesthesia, Euthanex Corp.) was employed to maintain the anesthesia status after the IP injection. The mouse was placed under a water tank to maximize the coupling efficiency of acoustic waves.

In order to acquire a 3D image, an imaging area of $4 \mathrm{~mm} \times 4 \mathrm{~mm}$ was scanned. In the original OR-PAM setup, step size in both lateral directions was $2.5 \mu \mathrm{m}$. 3D data set of $1600 \times 1600 \times 200$ was acquired in about 2 hours due to the slow mechanical scan and stage reposition during imaging in the original OR-PAM system. In the HSOR-PAM setup, step size in both lateral directions was $5 \mu \mathrm{m}$. 3D data set of $800 \times 800 \times 200$ was acquired in about 128 seconds with a 5000 $\mathrm{Hz}$ pulse repetition rate laser in the HSOR-PAM system.

\section{RESULTS}

In vivo, label-free mouse ocular anterior segment imaging with original OR-PAM system [6]

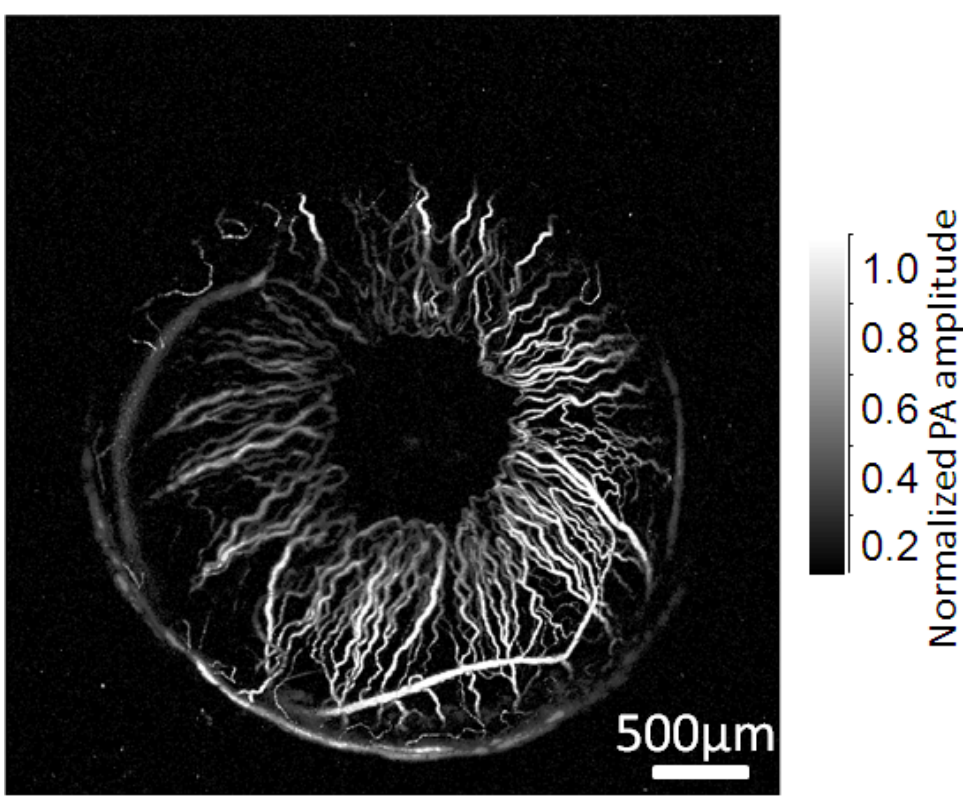

Fig 3. In vivo, label-free mouse ocular anterior segment imaging with the original OR-PAM system

Figure 3 demonstrated in vivo, label-free mouse ocular anterior segment imaging using the original OR-PAM system. Figure 3 is the MAP image of PA signals along depth direction. The system imaging resolution is $5 \mu \mathrm{m} \times 5 \mu \mathrm{m} \times 15 \mu \mathrm{m}$ in linear optical absorption range. However, its lateral imaging resolution could be better than the optical diffraction 
limit in the non-linear optical absorption range. Functional imaging was possible with multiple wavelength measurements [6]. The single pulse energy was set as $40 \mathrm{~nJ}$.

In vivo, label-free mouse ocular anterior segment imaging with HSOR-PAM system [7]

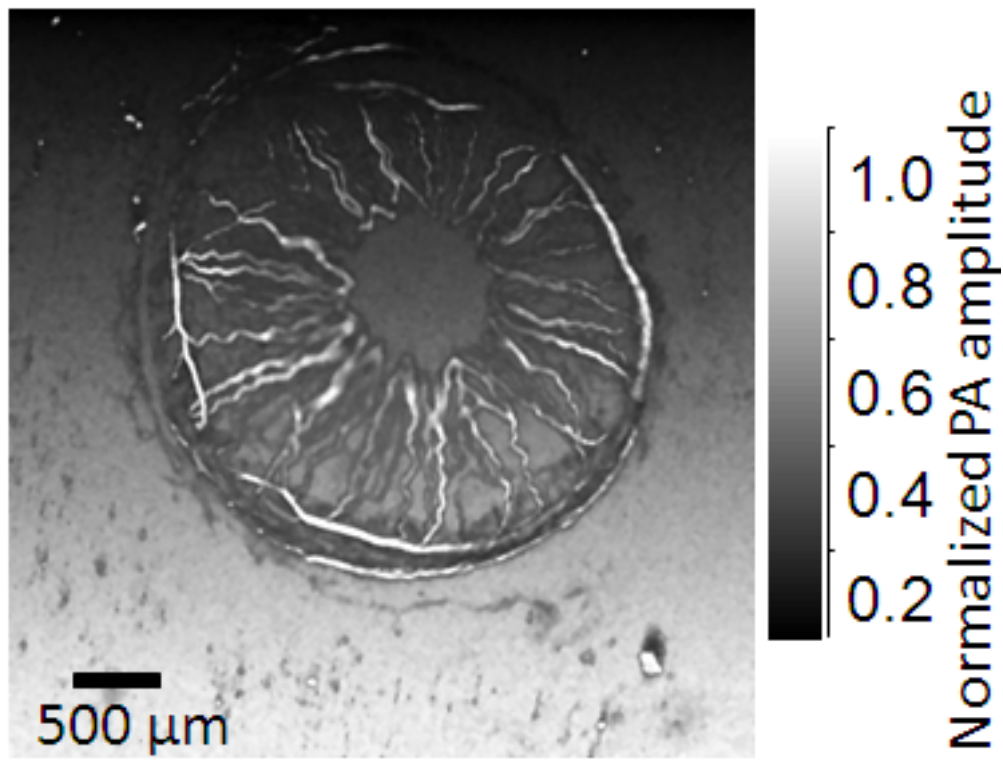

Fig. 4. In vivo, label-free mouse ocular anterior segment imaging using HSOR-PAM system

Figure 4 demonstrated in vivo, label-free mouse ocular anterior segment imaging using the fast HSOR-PAM [7] system. Figure 4 shows the 3D rendering result using the Amira software. The system imaging resolution is $3.5 \mu \mathrm{m} \times 3.5 \mu \mathrm{m} \times$ $52 \mu \mathrm{m}$. A higher frequency ultrasonic transducer could be used to improve the axial resolution easily. Figure 4 and Figure 3 were not acquired from the same mouse. The denser vasculature shown in Figure 3 is due to its higher SNR and denser scan $(2.5 \mu \mathrm{m}$ step size). The single pulse energy in the HSOR-PAM system was set as $60 \mathrm{~nJ}$.

\section{LASER SAFETY CONSIDERATIONS}

The major laser safety concern for the anterior segment of eye imaging is the safety of the anterior segment tissue instead of the retinal tissue [6]. We assume the laser focus is $200 \mu \mathrm{m}$ below the surface tissue. The surface illumination area $(\mathrm{NA}=0.1)$ is $30 \mu \mathrm{m}$.

In Figure 3, we scanned $4 \mathrm{~mm}$ using 1600 points. The step size was $2.5 \mu \mathrm{m}$. Thus the repetitive pulse number on the illumination area was 12 . The repetitive pulse limit is calculated as the following [8]:

$$
\mathrm{MPE}_{\mathrm{RP}}=\mathrm{n}^{-0.25} \times \mathrm{MPE}_{\mathrm{SP}}=12^{-0.25} \times 20 \mathrm{~mJ} / \mathrm{cm}^{2}=10.75 \mathrm{~mJ} / \mathrm{cm}^{2}
$$

After multiplying the $\mathrm{MPE}_{\mathrm{RP}}$ with surface illumination area, the maximum permissible pulse energy is calculated as 76 $\mathrm{nJ}$ per pulse. Thus our pulse energy of $40 \mathrm{~nJ}$ per pulse is within the safety limit.

In Figure 4, we scanned $4 \mathrm{~mm}$ using 800 points. The step size was $5 \mu \mathrm{m}$. Thus the repetitive pulse number on the illumination area was 6 . The repetitive pulse limit can be calculated as the following:

$$
\mathrm{MPE}_{\mathrm{RP}}=\mathrm{n}^{-0.25} \times \mathrm{MPE}_{\mathrm{SP}}=6^{-0.25} \times 20 \mathrm{~mJ} / \mathrm{cm}^{2}=12.78 \mathrm{~mJ} / \mathrm{cm}^{2}
$$

After multiplying the $\mathrm{MPE}_{\mathrm{RP}}$ with surface illumination area, the maximum permissible pulse energy is calculated as 90 $\mathrm{nJ}$ per pulse. Thus our pulse energy of $60 \mathrm{~nJ}$ per pulse is within the safety limit. 


\section{CONCLUSIONS}

We investigated the in vivo, label-free ocular anterior segment imaging with photo-acoustic microscopy (PAM) in mouse eyes. We demonstrated the unique advantage of endogenous contrast that is not available from both IFA and ICGA. The laser radiation was calculated to be within the ANSI laser safety limit. The in vivo, label-free nature of our imaging technology demonstrates its potential for ophthalmic applications. Future PAM technological development for in vivo imaging the anterior segment of human eyes is desirable.

\section{ACKNOWLEDGEMENT}

The mouse ocular anterior segment imaging demonstration done on the original OR-PAM system was contributed in equal measure by Bin Rao and Song Hu. The HS-ORPAM system and mouse ocular anterior segment imaging demonstration were contributed in equal measure by Bin Rao and Li Li. The research was supported by the National Institutes of Health grants R01 EB000712, EB000712A2S1, R01 EB00071207S2, R01 EB008085, R01 CA113453901, U54 CA136398, and 5P60 DK02057933. L.W. has a financial interest in Microphotoacoustics, Inc. and Endra, Inc., which, however, did not support this work.

\section{REFERENCES}

[1] Rosario Brancato, Francesco Bandello, and Rosangela Lattanzio, "Iris Fluorescein Angiography in Clinical Practice," Survey of Ophthalmology, 42, 41-70(1997).

[2] Yasuhiro Maruyama, Shoji Kishi, Yo Kamei, Ryo Shimizu, and Yasutaka Kimura, "Infrared Angiography of the Anterior Ocular Segment," Survey of Ophthalmology, 39, S40-48(1995).

[3] K. Maslov, H. F. Zhang, S. Hu, and L. V. Wang, "Optical-resolution photoacoustic microscopy for in vivo imaging of single capillaries," Optics Letters 33, 929-931 (2008).

[4] L. V. Wang, "Prospects of photoacoustic tomography," Medical Physics 35 (12), 5758-5767 (Dec. 2008).

[5] Lihong Wang, "Multiscale photoacoustic microscopy and computed tomography," Nature Photon. 3, 503509(2009).

[6] Song Hu, Bin Rao, Konstantin Maslov, and Lihong V. Wang, "Label-free photoacoustic ophthalmic angiography," Opt. Lett. 35, 1-3 (2010).

[7] Bin Rao, Li Li, Konstantin Maslov, and Lihong V. Wang, "Hybrid-scan optical-resolution photoacoustic microscopy," (to be submitted to OL).

[8] Laser Institute of America, American National Standard for Safe Use of Lasers ANSI Z136.1-2000 (American National Standards Institute, Inc., 2000). 